\title{
$\mathrm{Au}$ 单原子的制备及其在 $\mathrm{CO}$ 氧化反应中荷电状态的表征
}

张涛

中国科学院大连化学物理研究所, 催化基础国家重点实验室, 辽宁大连 116023

\section{Preparing Au Single-Atoms and Unraveling Their Charge State during CO Oxidation}

\section{ZHANG Tao}

State Key Laboratory of Catalysis, Dalian Institute of Chemical Physics, Chinese Academy of Sciences, Dalian 116023, Liaoning Province, P. R. China.

Email: taozhang@dicp.ac.cn

(a)

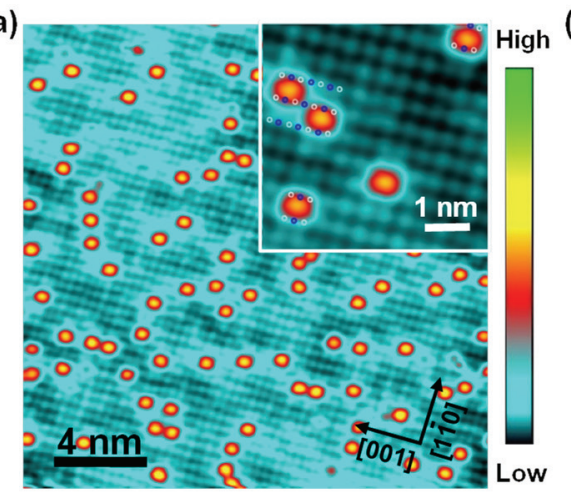

(c)

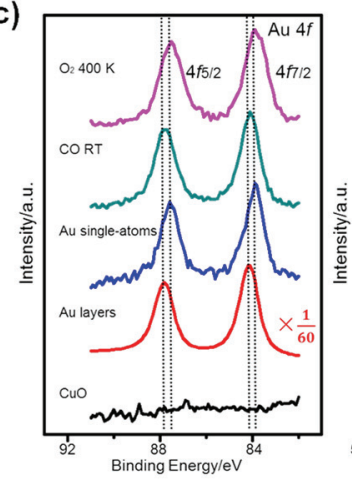

(b)

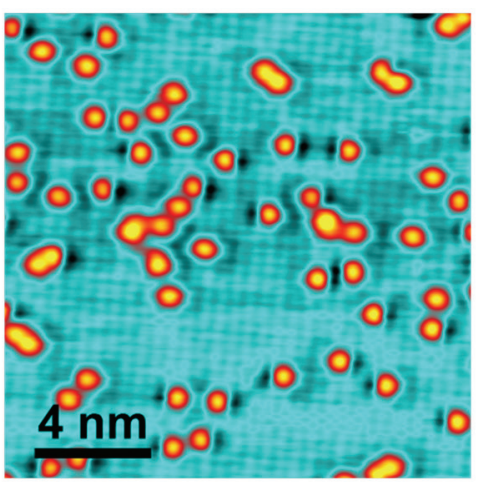

(d)

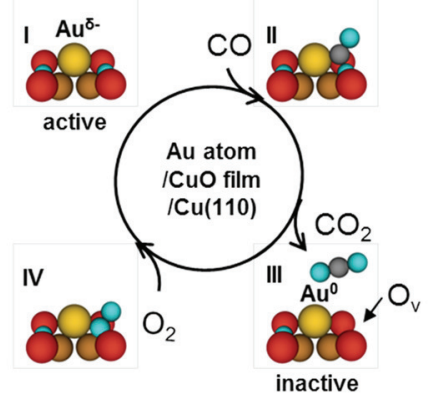

(a) 单层 $\mathrm{CuO}$ 薄膜上制备的 $\mathrm{Au}$ 单原子 STM 图。(b) $\mathrm{CO}$ 分子在室温条件下与 $\mathrm{Au}$ 单原子近邻晶格氧形成的 氧空穴 STM 图。(c) CO 氧化反应过程中 Au $4 f$ 和 O $1 \mathrm{~s}$ XPS 信号的变化, 其中通入 CO 为室温, 通入 $\mathrm{O}_{2}$ 时温度为 $400 \mathrm{~K}$ 。(d) $\mathrm{Au}$ 单原子活化的 $\mathrm{CO}$ 氧化反应机理图。

一般来说, 相比传统催化剂, 高分散的纳米催 化剂的活性大大增强, 而负载型金属催化剂分散 的极限是金属以单原子的形式均匀分布在载体
上, 这不仅是负载型金属催化剂的理想状态, 而且 也将催化科学带入到一个更小的研究尺度一单原 子催化 ${ }^{1,2}$ 。单原子催化是多相催化领域的新概念, 
现已成为催化领域新的前沿与热点之一。单原子 催化剂兼具均相催化剂的 “孤立活性位点” 和多相 催化剂 “稳定易分离” 的特点, 因而有望开辟均相 催化剂多相化的新途径, 成为联结均相与多相催 化的桥梁 ${ }^{3}$ 。在传统催化中金 $(\mathrm{Au})$ 为惰性金属, 在 高分散情况下则表现出特定反应的高活性 4,5 。如何 解释高分散 $\mathrm{Au}$ 的高活性来源也一直是催化领域的 研究热点, 其中 $\mathrm{Au}$ 的荷电状态受到广泛关注 ${ }^{6}$, 然 而在反应过程中 $\mathrm{Au}$ 的荷电状态的变化还没有得 到系统的研究。

最近, 北京大学化学与分子工程学院吴凯教 授课题组通过调控氧化物表面自由能的方法实现 了 $\mathrm{Au}$ 单原子制备, 并考察了其在一氧化碳 $(\mathrm{CO})$ 氧 化反应中荷电状态的演化过程及相应反应活性的 变化。一般情况下金属相对于氧化物具有高表面 自由能, 于是在氧化物表面自由生长的金属会倾 向于聚集。而当氧化物变为薄膜生长在块体金属 表面时, 氧化物表面受到块体金属电子效应的影 响, 其表面自由能也相应增大, 于是此时沉积的金 属就有可能处于高分散状态 ${ }^{7}$ 。吴凯教授课题组使 用在 $\mathrm{Cu}(110)$ 单晶表面生长的单层 $\mathrm{CuO}$ 薄膜作为 载体, 在 0.05 单层覆盖度情况下得到了高分散高 稳定性的的 $\mathrm{Au}$ 单原子。该 $\mathrm{Au}$ 单原子在 $400 \mathrm{~K}$ 温 度条件下依旧稳定存在。该单原子制备方法简单 易行, 不需要通过吸附分子或者嵌入晶格来稳定 单原子, 是制备单原子模型催化剂的新思路。

使用该 $\mathrm{Au}$ 单原子作为模型催化剂, 吴凯课题 组系统考察了其在 $\mathrm{CO}$ 氧化反应中的机理。在反 应前, $\mathrm{Au}$ 单原子为负电状态。主要途径是 $\mathrm{CuO}$ 中 的近邻氧 $(\mathrm{O})$ 离子将电子传递给 $\mathrm{Au}$, 从而活化了近 邻 $\mathrm{O}$ 离子, 同时负电状态的 $\mathrm{Au}$ 单原子有助于金 属和 $\mathrm{CO}$ 间的 $d-\pi^{*}$ 反馈, 从而得以活化吸附的 $\mathrm{CO}$ 分子。这样 $\mathrm{CO}$ 分子跟近邻 $\mathrm{O}$ 离子在室温条件下 反应生成 $\mathrm{CO}_{2}$ 分子, 而 $\mathrm{O}$ 离子处产生 $\mathrm{O}$ 空穴。当 反应发生后, $\mathrm{Au}$ 单原子则变为电中性, 此时反应 活性丧失。当使用 $\mathrm{O}_{2}$ 在 $400 \mathrm{~K}$ 修补晶格 $\mathrm{O}$ 缺陷
后, $\mathrm{Au}$ 单原子又变为负电状态, 恢复了反应活性。 由此构成一个完整的反应循环, 遵从 $\mathrm{MvK}$ 反应机 理。该研究利用了扫描隧道显微镜(STM)的超高空 间分辨技术和 $\mathrm{X}$ 射线光电子能谱(XPS)的化学分 辨能力, 在原子尺度上清晰地表征了 $\mathrm{Au}$ 单原子和 氧化物载体离子在反应过程中的荷电状态演化过 程及其与反应活性的关联, 这为解释高分散 $\mathrm{Au}$ 的 高活性提供了重要依据。

该研究工作近期已在 Journal of the American Chemical Society 上在线发表 ${ }^{8}$ 。该研究与中国科 学院大连化学物理研究所杨学明院士, 新加坡国 立大学化学系陈伟教授, 中国科学技术大学邵翔 教授课题组合作完成。

\section{References}

(1) Qiao, B.; Wang, A.; Yang, X.; Allard, L. F.; Jiang, Z.; Cui, Y.; Liu, J.; Li, J.; Zhang, T. Nat. Chem. 2011, 3, 634. doi:10.1038/nchem.1095

(2) Yang, X. F.; Wang, A.; Qiao, B.; Li, J.; Liu, J.; Zhang, T. Acc. Chem. Res. 2013, 46, 1740. doi:10.1021/ar300361m

(3) Lang, R.; Li, T.; Matsumura, D.; Miao, S.; Ren, Y.; Cui, Y. T.; Tan, Y.; Qiao, B.; Li, L.; Wang, A.; et al. Angew. Chem. Int. Ed. 2016, 55, 16054. doi:10.1002/anie.201607885

(4) Haruta, M.; Kobayashi, T.; Sano, H.; Yamada, N. Chem. Lett. 1987, 2, 405. doi: 10.1246/cl.1987.405

(5) Hutchings, G. J.; Joffe, R. Appl. Catal. 1986, 20, 215. doi:10.1016/0166-9834(86)80017-3

(6) Yoon, B.; Häkkinen, H.; Landman, U.; Wörz, A. S.; Antonietti, J. M.; Abbet, S.; Judai, K.; Heiz, U. Science 2005, 307, 403. doi:10.1126/science. 1104168

(7) Zhou, X.; Yang, W.; Chen, Q.; Geng, Z.; Shao, X.; Li, J.; Wang, Y.; Dai, D.; Chen, W.; Xu, G.; et al. J. Phys. Chem. C 2016, 120, 1709. doi:10.1021/acs.jpcc.5b11362

(8) Zhou, X.; Shen, Q.; Yuan, K.; Yang, W.; Chen, Q.; Geng, Z.; Zhang, J.; Shao, X.; Chen, W.; Xu, G.; et al. J. Am. Chem. Soc. 2018, doi: $10.1021 /$ jacs. 7 b10394 